\title{
Measuring Geometrical Parameters of Involute Spur Gears to Sub-pixel Resolution.
}

\author{
Mark J Robinson * John P Oakley \\ Dept. of Electrical Engineering \\ University of Manchester \\ Manchester M13 9PL \\ email mark-r(̣̂spec0.ee.man.ac.uk
}

\begin{abstract}
The problem of accurate determination of the position, orientation and width of involute spur gear teeth using a single front-lit image is addressed. The information is used to compute global measures of tooth concentricity and pitch variations across a gear wheel. The approach is based on the use of specialised filters which make maximum use of the grey level information available in the image. Tooth location consistent to \pm 0.04 pixels has been achieved in realistic images.
\end{abstract}

\section{Introduction}

Gears are manufactured to very close tolerance, better than $10 \mu \mathrm{m}$ for high quality components ([1], [2]). The current methods of testing these tolerances is to use co-ordinate measuring machines, or a shadowgraph (an optical projection system which casts a magnified shadow of the tooth onto a calibrated screen). Both these techniques have inherent disadvantages; the former is expensive while the latter is time consuming. Contact with industry has revealed that there would be demand for a reliable and low-cost automatic inspection system based around image analysis. Measurement using image analysis is difficult, however. because the high precision required suggests that a "close-up" image of each tooth should be used, but the need to measure in a global coordinate system would then require accurate registration of a number of these sub-images. This implies that an array of cameras, or a mechanical system to move the part under a single camera, would be required. An image analysis system which uses one image would be simpler, cheaper and more reliable.

The aim of this study is to determine the measurement accuracy which is possible in this type of application from a single image.

- Funded by the SERC 


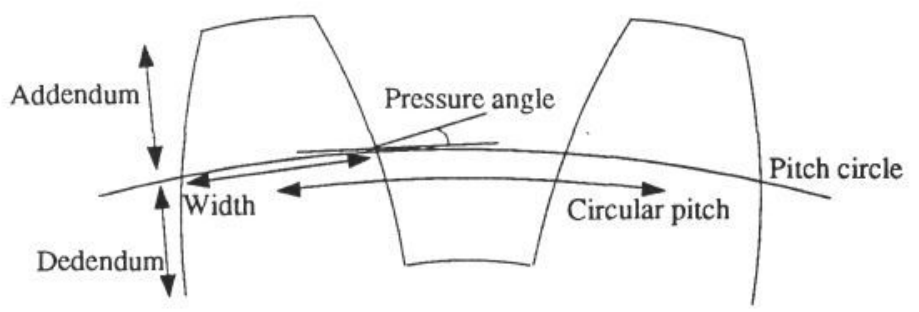

Figure 1: A gear profile

The definitions of dimensions associated with a gear tooth are shown in figure 1. The sides of the teeth must accurately match a well defined curve called an involute to a circle. A complete inspection system would need to measure

- Concentricity of the teeth.

- Error in the pitch of the teeth.

- Error in the involute profile and pressure angle.

The system discussed here takes an initial estimate of the position of a tooth from a cue generator. and iteratively refines it to return the position of the centre of the tooth and its orientation. in a global co-ordinate system, and the width of the tooth across the pitch circle.

In [3] we show that a specialised filter, the Curved Iterative Boundary Locator (CIBL), makes more effective use of image information than model fitting procedures based on edge data. When applied to the gear images, however, the small length of arailable boundary and the inaccurate match between the filter shape and the boundary shape led to two difficulties. The first was that the final position of the operator was affected by changes in grey level along the tooth boundary. Also, the operator was not completely reliable in that highlights on the tooth could cause the operator to align itself with the top of the tooth rather than the side. even when the initial position was very close to the required boundary:

This disappointing result led us to define more specialised operators for this particular visual task. These operators, CIBL-2, CIBL-3 and INVOLUTE-2 are described in the following sections.

\section{CIBL-2 and CIBL-3}

A new operator, CIBL-2, was developed to make use of prior knowledge of the structure of the gear tooth. This was based on the sum of two CIBL filters, one for each side of the tooth. The filters were free to move and rotate as a unit, but their orientation was fixed relative to each other. The position, orientation and separation of these filters were varied by an optimisation routine to maximise the filter output. 


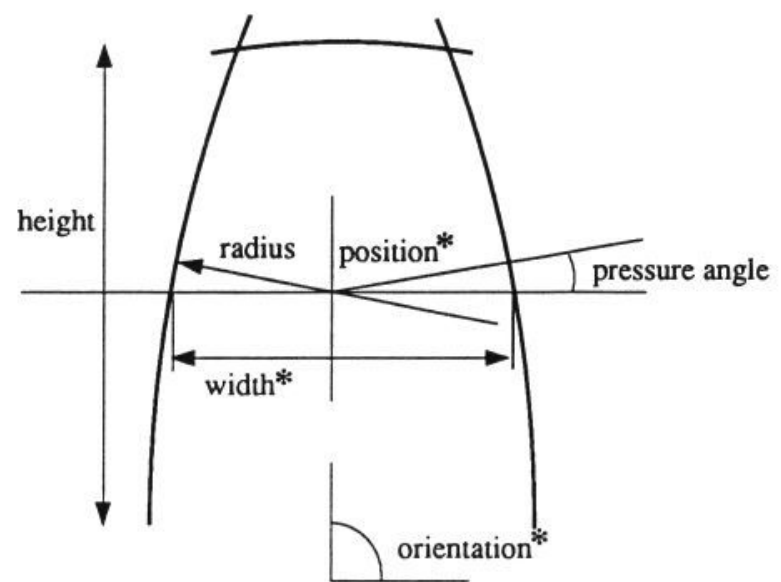

Figure 2: The composite operator. Parameters marked * are under the control of the optimisation routine.

This led to a significant improvement over the single filter, but it was still easily confused by highlights on the edges of teeth, where one of the filters would centre itself on the highlight. In order to counteract this problem a third filter was introduced to the operator, aligned with the outside edge of the tooth. This filter's output was weighted so as to keep the operator locked onto a tooth, while not dominating the operator output. The geometry and parameters of this operator, CIBL-3, are shown in figure 2.

Although this operator worked well, its performance is compromised by a number of factors, the choice of filter radius to give the best approximation to the required involute is not obvious, and the need for the operator to use the outer (non-contacting) surface of the tooth is undesirable because this face is not subject to any critical tolerance specification.

\section{INVOLUTE-2}

To circumvent these problems, a new filter which modelled the involute profile of the tooth more closely was developed.

The parametric equations for the involute to a circle of radius $a$, starting from the origin, are

$$
\begin{aligned}
& x_{i}=a(\cos t+t \sin t-1) \\
& y_{i}=a(\sin t-t \cos t)
\end{aligned}
$$

This curve is shown in figure 3 .

The distance, $r(t)$, of a general point $\left(x_{p}, y_{p}\right)$ from this involute is given by

$$
r(t)^{2}=\left(x_{i}-x_{p}\right)^{2}+\left(y_{i}-y_{p}\right)^{2}
$$




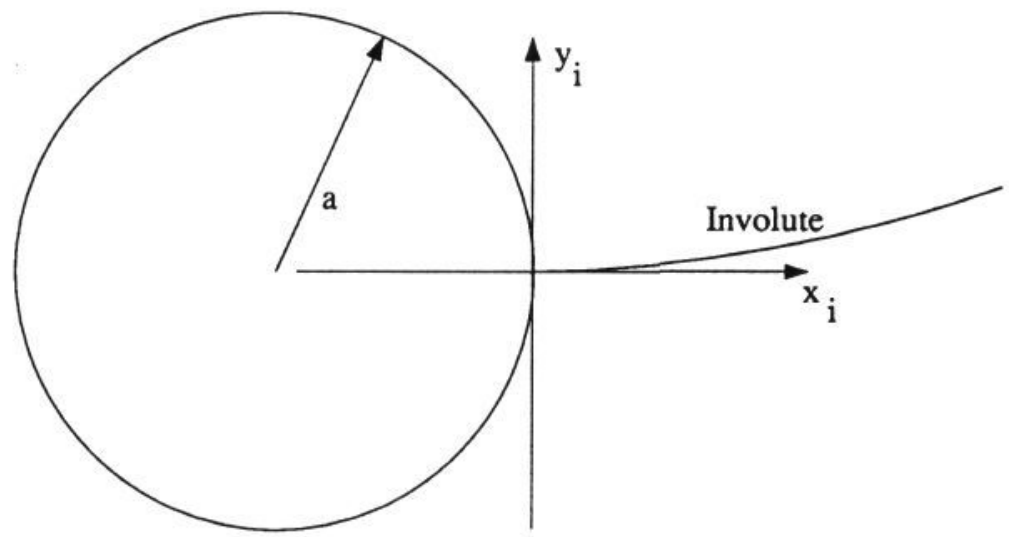

Figure 3: The involute to a circle of radius $a$.

This distance is minimised when

$$
\frac{\partial r(t)^{2}}{\partial t}=0
$$

which implies that

$$
y_{p} \sin t+\left(x_{p}+a\right) \cos t-a=0
$$

Solving 4 gives $t_{\min }$, the value of $t$ at which the involute passes closest to $\left(x_{p}, y_{p}\right)$ and by substituting into 1 we can find $r\left(t_{\min }\right)$, the minimum distance. The basic gaussian filter is then defined by

$$
f=\exp \left(\frac{r\left(t_{\min }\right)^{2}}{\sigma_{d}^{2}}+\frac{p^{2}}{\sigma_{p}^{2}}\right)
$$

where $\sigma_{d}$ and $\sigma_{p}$ are the filter extent in the detector and projector directions respectively, and the projector function $p$ is defined as

$$
p=\left(x_{p}-x_{c e n}\right)^{2}
$$

which assumes that the filter is approximately straight. $x_{c e n}$ is then the distance of the peak in the projector Gaussian function from the origin, $3 \sigma_{p}$ is a reasonable value. A typical filter defined in this way is shown in figure 4 .

The derivative of this filter normal to the involute is used in the operator, and it is moved to its required position by applying an affine transform to it.

The INVOLUTE-2 operator was built using two of these filters, one to model each side of the tooth. Again, the filters are free to move and rotate as a unit, and to move apart to fit the width of the tooth.

The partial derivatives of the operator required by the optimisation routine were estimated using finite differences because of the complexity of these functions. 


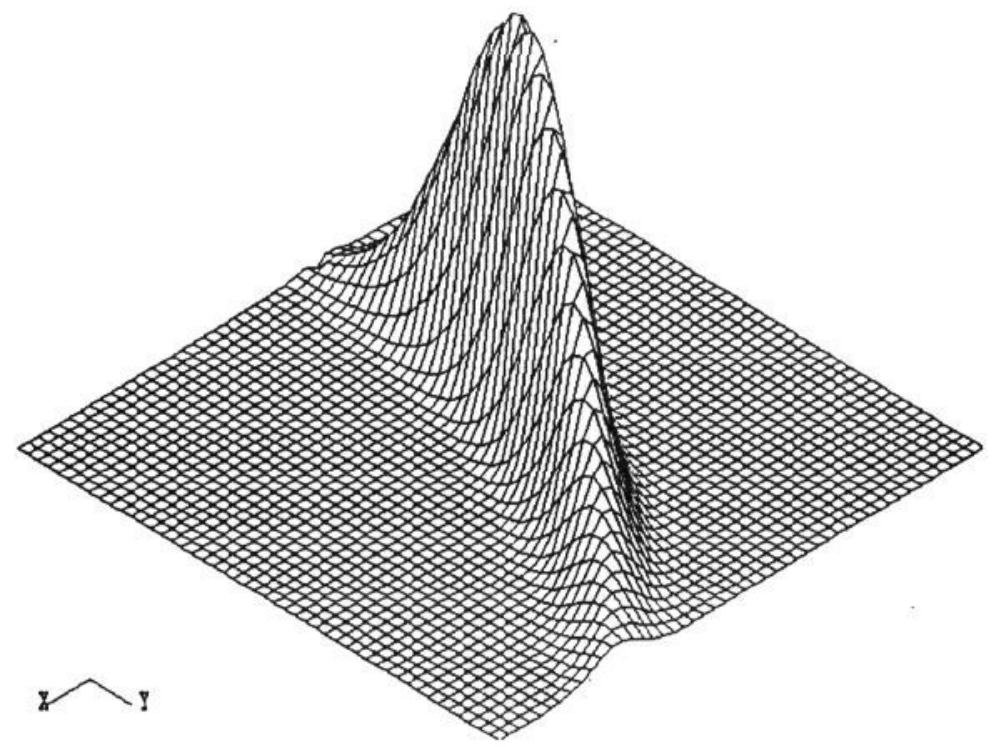

Figure 4: A typical involute filter shape.

\section{Testing}

Figure 5 shows part of an image of a gear wheel, magnified to show a single tooth, with the final position of the operator overlayed, for both the CIBL-3 and INVOLUTE-2 operators.

The gear locators were tested by measuring the distance between the centres of three adjacent teeth in six images of the same gear wheel. A tooth occupied an area of around $40 \mathrm{by} 40$ pixels, where one pixel corresponded to about $0.1 \mathrm{~mm}$. In each successive image the gear wheel was moved very slightly, and the direction of the lighting changed, in order to make the images as different as possible without affecting the geometry. The values of distance measured should be invariant between the images, but changes in noise and shading result in discrepancies in the position which maximises the operator output.

The RMS variation between the results was used as an indication of the random error in the operator, although systematic errors cannot be quantified without a calibrated reference.

The results for both operators are summarised in table 1 .

It can be seen that both operators produced similar results. of around 0.04 pixels, which corresponds to $4 \mu \mathrm{m}$. The INVOLUTE-2 operator is still affected by highlights and noise to some extent. although it is considerably better than CIBL-2. To improve the performance of INVOLUTE-2, a cost function based on the expected position of the base circle or outer surface of the tooth could be included. Requesting an accuracy better than around 0.005 pixels caused the optimisation routine to fail. or return a very inconsistent result, implying 

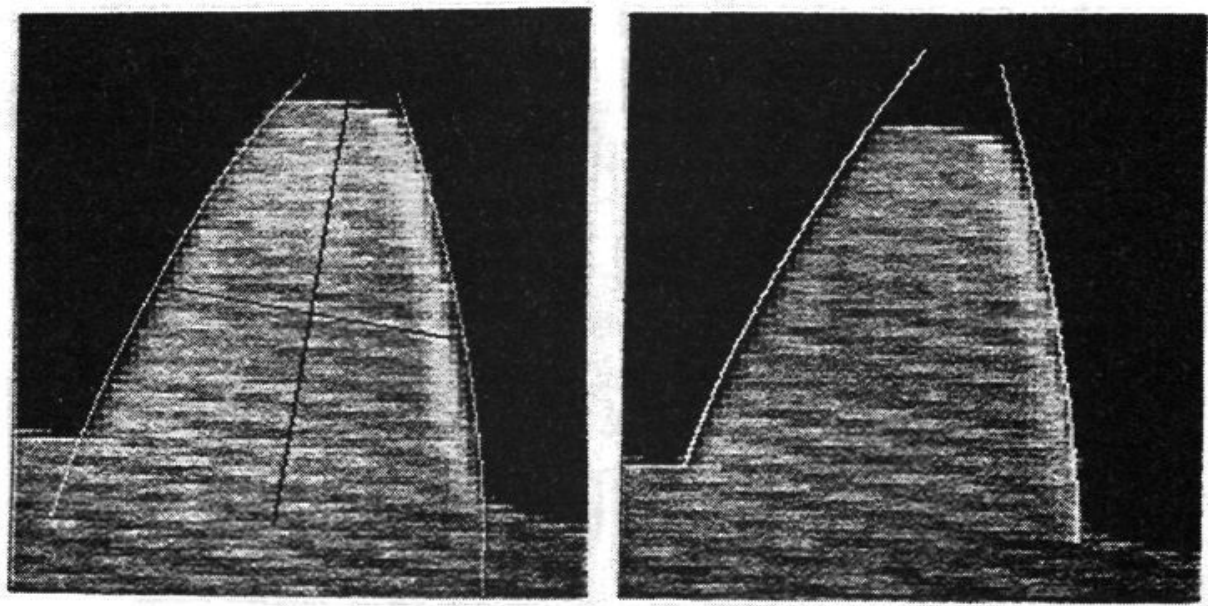

Figure 5: Magnified views of the final tooth position measured by (a) the CIBL-3 operator, and (b) the INVOLUTE-2 operator.

that this is the limit of accuracy available with these test images.

\section{Conclusion}

Results from the initial trials of the tooth locator are very encouraging, achieving the accuracy required for even the closest tolerance gears from a single image. Obviously the final accuracy achievable by the system will depend on the camera calibration, but the requirements of the calibration procedure and image acquisition system are greatly simplified by the ability to work from a single image.

There are other limitations on the accuracy available from the system apart from the image analysis algorithm, particularly from the camera optics, and this is an area currently under investigation.

\section{Acknowledgements}

The authors would like to acknowledge the assistance of the following companies, who provided useful information about the gear manufacturing process and samples of their products.

- Newmont Engineering Co. Ltd.

- Portescap UK Ltd.

- S.H. Muffett Ltd.

- Spline Gauges Ltd.

- University of Newcastle.

The project is funded by the SERC. 


\begin{tabular}{|c|c|c|c|c|c|}
\hline \multicolumn{3}{|c|}{ CIBL-3 } & \multicolumn{3}{|c|}{ INVOLUTE-2 } \\
\hline \multicolumn{2}{|c|}{ distance (pixels) } & \multirow{2}{*}{$\begin{array}{l}\text { error } \\
\text { (pixels) }\end{array}$} & \multicolumn{2}{|c|}{ distance (pixels) } & \multirow{2}{*}{$\begin{array}{l}\text { error } \\
\text { (pixels) }\end{array}$} \\
\hline image 1 & image 2 & & image 1 & image 2 & \\
\hline 60.4003 & 60.3819 & 0.0184 & 62.7561 & 62.6994 & 0.0567 \\
\hline 60.3819 & 60.3943 & 0.0124 & 62.6994 & 62.6988 & 0.0006 \\
\hline 60.3843 & 60.4022 & 0.0179 & 62.6988 & 62.6564 & 0.0423 \\
\hline 60.3922 & 60.4003 & 0.0081 & 62.6664 & 62.7344 & 0.0670 \\
\hline 60.4003 & 60.4334 & 0.0330 & 62.7344 & 62.7629 & 0.0285 \\
\hline 60.7703 & 60.7923 & 0.0220 & 60.8294 & 60.8709 & 0.0415 \\
\hline 60.7923 & 60.8059 & 0.0135 & 60.8709 & 60.8548 & 0.0161 \\
\hline 60.8059 & 60.9102 & 0.1043 & 60.9548 & 60.9072 & 0.0476 \\
\hline 60.9102 & 60.8829 & 0.0273 & 60.9072 & 60.8539 & 0.0532 \\
\hline 60.8829 & 60.8983 & 0.0155 & 60.8539 & 60.8981 & 0.0442 \\
\hline$\overline{\mathrm{RM}}$ & error $=$ & 035 & $\overline{\mathrm{RN}}$ & error = & .040 \\
\hline
\end{tabular}

Table 1: Summary of test results.

\section{References}

[1] British Standard 436 part 2 The British Standards Institute 1970

[2] British Standard 4582 part 1 The British Standards Institute 1970

[3] Robinson M J, Oakley J P and Shann R T. An efficient and Robust Local Boundary Operator. Proceedings of the BMVC 1991. 